Supporting Information

\title{
Controlling Microbial Dynamics through Selective Solute Transport Across Functional Nanocultures
}

\author{
Shanna-Leigh Davidson ${ }^{1}$, Tagbo H.R. Niepa ${ }^{1,2,3,4,5,6 *}$ \\ ${ }^{1}$ Department of Chemical and Petroleum Engineering, ${ }^{2}$ Department of Bioengineering; ${ }^{3}$ Department of \\ Civil and Environmental Engineering; ${ }^{4}$ Department of Mechanical Engineering and Materials Science; \\ ${ }^{5}$ Center for Medicine and the Microbiome, ${ }^{6}$ McGowan Institute for Regenerative Medicine, University of \\ Pittsburgh, Pittsburgh, United States
}

*Corresponding author: tniepa@pitt.edu

Keywords: microbial dynamics, microfluidics, nanocultures, poly(dimethylsiloxane), chemical functionalization, Flory-Huggins.

This supplementary information provides evidence for supporting experiments complementary to the research article. Here, we have included a detailed discussion on finding Flory-Huggins interaction parameters for solute-polymer pairs. We further include bacterial growth curves to show inhibitory behavior of selected antimicrobial molecules. We show optimization of growth of bacteria in minimal media to determine successful growth in selected media. Figures complementary to sugar diffusion have also been included.

\section{Determination of Flory-Huggins Interaction Parameter}

Solubility between two molecules is often described by the cohesive energy density (CED) of each molecule, a value associated with the intermolecular attractive forces per unit volume of material. Subsequently, solubility occurs when two materials exhibit similar CEDs, such that the attractive intermolecular forces are overcome, and solvation may occur. The CED can be further described by the total solubility parameter, or Hildebrand value (Equation 1): 


$$
\delta=C E D^{\frac{1}{2}}=\left(-\frac{U}{V}\right)^{\frac{1}{2}}
$$

where $\mathrm{U}$ is the molar internal energy $(\mathrm{cal} / \mathrm{mol})$ and $\mathrm{V}$ is the molar volume $\left(\mathrm{cm}^{3} / \mathrm{mol}\right)$. The Hildebrand solubility parameter has been extensively used to predict polymer solubility (or swelling behavior) in nonpolar solvents with great success. ${ }^{1-3}$ Subsequently, attempts have recently been made to investigate the applicability of using Hildebrand solubility parameters to predict the solubility behavior of binary and multicomponent polymer-drug solution. ${ }^{4-8}$ In these cases, the Flory-Huggins solution theory has been used with modifications to describe the Gibbs free energy of a drug-polymer binary system; the theory of which has been discussed extensively elsewhere. 1, 9, 10 The Flory-Huggins interaction parameter between drug and polymer thus arises out of the equation for Gibbs free energy, and provides an estimation of whether a solute will preferably partition into the polymer phase or not. The Flory-Huggins interaction parameter, $\chi$, depends on the solubility parameters, $\delta$, of both the solute (component 1 ) and polymer (component 2 ) as in the relationship (Equation 2):

$$
\chi=\frac{\mathrm{V}_{1}\left(\delta_{1}-\delta_{2}\right)^{2}}{\mathrm{RT}}
$$

where $\mathrm{V}_{1}$ is the molar volume of component $1, \mathrm{R}$ is the real gas constant in appropriate units, and $\mathrm{T}$ is the absolute temperature. A small interaction parameter typically indicates solubility; when $\delta_{1}$ and $\delta_{2}$ are similar, "like dissolves like" and it is predicted that the solute will partition and permeate through the polymer.

Although many advances have been made to understand relative interactions between drug-polymer blends, it is difficult to predict the solubility behavior of these systems because solubility parameters are not commonly known for molecules of biological interest, such as antimicrobials and the fluorescent dyes chosen for this study. Whereas solubility parameters for low molecular weight liquids can be conveniently found experimentally by obtaining the heat of vaporization, such direct methods do not work for high molecular weight polymers and crystal powders due to their low volatility. Hence, a common indirect method for estimating $\delta$ for such materials is based on Fedor's group contribution “molar-attraction constants" method, whereby only the chemical structure of the material is needed to sum the molar attractions of each functional group. Since the development of the group contributions method, it has evolved through many iterations to become an accurate tool in estimating several thermodynamic properties of compounds. ${ }^{11-14}$ 
One crucial enhancement to the applicability of Hilderbrand's total solubility parameter was the development of Hansen's partial solubility parameters, which better describe the different intermolecular forces governing a molecule. It is now widely understood that three kinds of intermolecular forces exist: dispersive, polar and hydrogen-bonding forces, all of which play an integral role in the thermodynamic properties of materials. Thus, the total solubility parameter, $\delta_{t}$, is expanded upon as in Equation 3 :

$$
\delta_{\mathrm{t}}=\sqrt{\delta_{\mathrm{d}}^{2}+\delta_{\mathrm{p}}^{2}+\delta_{\mathrm{h}}^{2}}
$$

where $\delta_{\mathrm{d}}$ represents dispersive forces, $\delta_{\mathrm{p}}$ represents polar forces and $\delta_{\mathrm{h}}$ describes hydrogen bonding forces. Accounting for these three forces results in a significantly more accurate estimate for the total solubility parameter and subsequently the predictive power of the Flory-Huggins interaction parameter has larger capacity.

Solubility parameters are subsequently used in the calculation of Flory-Huggins interaction parameters to predict miscibility of the solute in the polymer. For miscibility to remain thermodynamically favorable, $\chi$ must be small; however, the theoretical critical threshold for solubility is system specific, dependent on how the polymer volume lattice is defined. ${ }^{15}$ All values calculated for the solute-nanoculture system have been reported in Table 1 in the research article.

\section{Antibiotic-induced growth inhibition of $E$. coli cells}

Escherichia coli cells used for this study were tested for susceptibility to selected antimicrobials. For all antibiotic molecules, bar ampicillin, E. coli DH5- $\alpha$ pNCS-mClover $3\left(\operatorname{amp}^{\mathrm{R}}\right)$ (E. coli GFP) was the selected bacterial strain. For ampicillin specifically, E. coli Nissle pRSH103 RFP $\left(\right.$ tet $\left.^{\mathrm{R}}\right)($ E. coli RFP) was the selected bacterial strain. In all cases, bacteria were grown in $200 \mu \mathrm{L}$ UFTYE media in the presence of antibiotic molecules in a 96-well plate assay for 48 hours at $37^{\circ} \mathrm{C}$ in aerobic conditions. Absorbance was measured every 10 minutes at $\mathrm{OD}_{600}$ using a 96-well plate reader (Cytation 5 imaging reader, BioTek Instruments Inc.) Antibiotic molecules ampicillin, chloramphenicol, ofloxacin and tetracycline were tested at concentrations ranging from 0 to $75 \mu \mathrm{M}$, and hydrogen peroxide was tested from 0-64 mM (Figure S1). In all cases, E. coli proved susceptible to the antibiotics at higher tested concentrations, and therefore, high cell fluorescence measurements within nanocultures are indicative of impermeability of the drug through the polymer shell. 


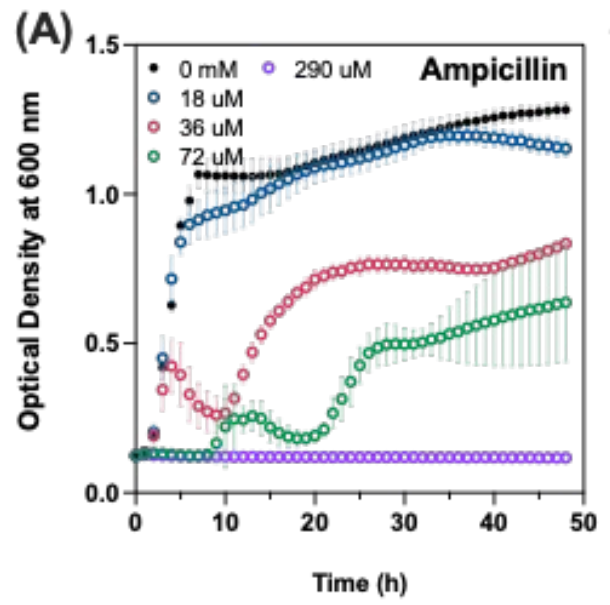

(B)

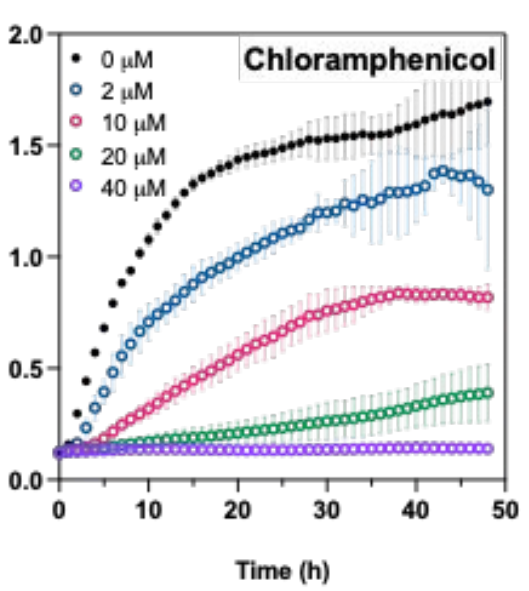

(E)

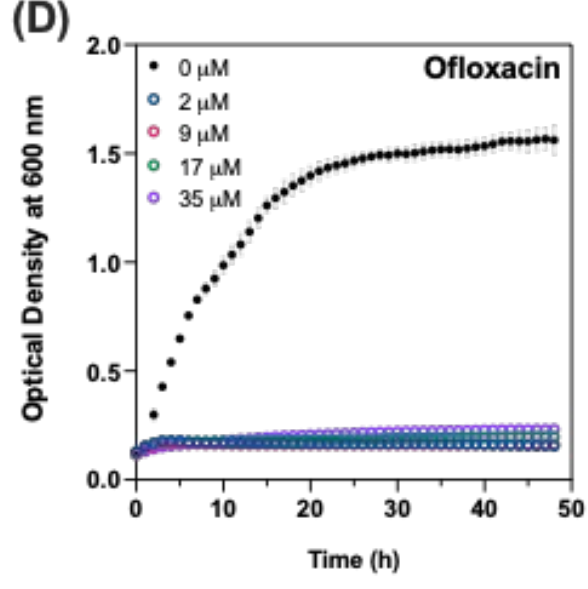

(C)

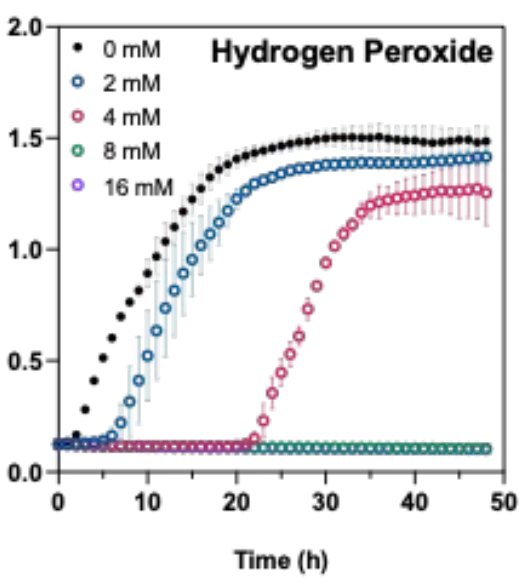

Figure S1. Growth inhibition curves with antimicrobials. (A) E. coli Nissle pRSH103 RFP (tet $\left.{ }^{\mathrm{R}}\right)($ E. coli RFP) tested for susceptibility to ampicillin for diffusion test. E. coli mClover $3\left(\mathrm{amp}^{\mathrm{R}}\right)($ E. coli GFP) was tested against (B) chloramphenicol; (C) hydrogen peroxide; (D) ofloxacin; and (E) tetracycline in concentrations ranging from $0-200 \mu \mathrm{g} / \mathrm{mL}$, to determine the minimum inhibitory concentration of each of antimicrobial. Hydrogen peroxide was tested in concentrations ranging from 0-64 mM. Growth inhibition of cells was achieved in each case, and appropriate antimicrobial concentrations were then selected for diffusion tests.

\section{Controls for fluorescent probes}

To ensure that the fluorescent dyes were all working as intended to dye the bacterial cells within the nanocultures, cells that were not encapsulated were stained as per manufacturer's protocols. Escherichia coli Nissle WT (non-fluorescent wild type) cells were allowed to adhere to glass cover slips and were then stained with the fluorescent stains: crystal violet $(0.5 \% \mathrm{v} / \mathrm{v})$, Nile blue $(25 \mu \mathrm{M})$, Nile red $(25 \mu \mathrm{M})($ Figure S2), and Acridine Orange $(15 \mu \mathrm{M})$ (Figure S3). Cells were incubated with the fluorescent dyes for 30 mins at room temperature and were then imaged at 50× under brightfield and fluorescent channels. 


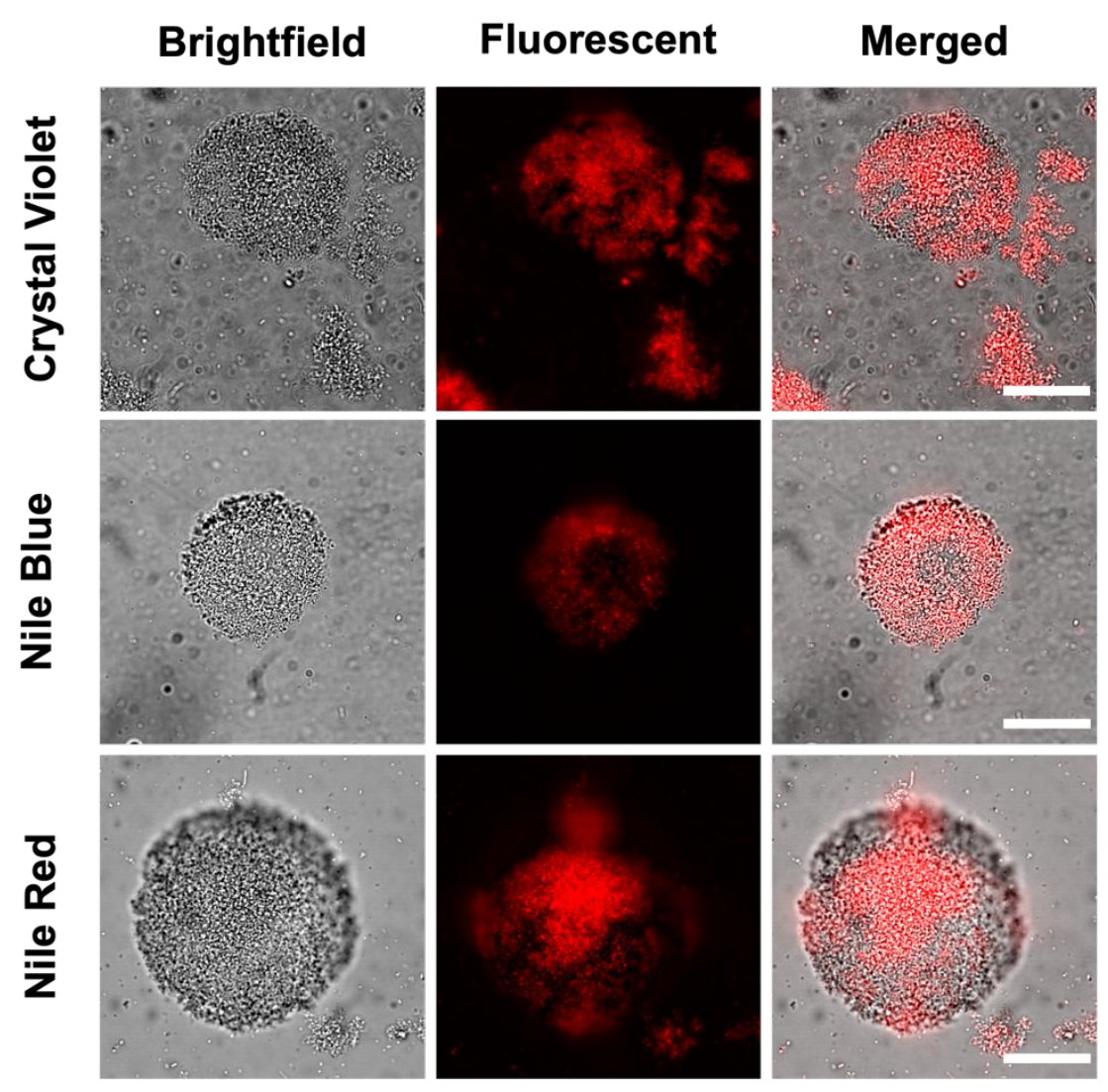

Figure S2. Controls for fluorescent probe staining of E. coli cells. Fluorescent and brightfield images taken at $50 \times$ show that crystal violet $(0.5 \%)$, Nile blue and Nile red $(25 \mu \mathrm{M})$ readily stain nonencapsulated cells. Scale bar: $50 \mu \mathrm{m}$.

Brightfield

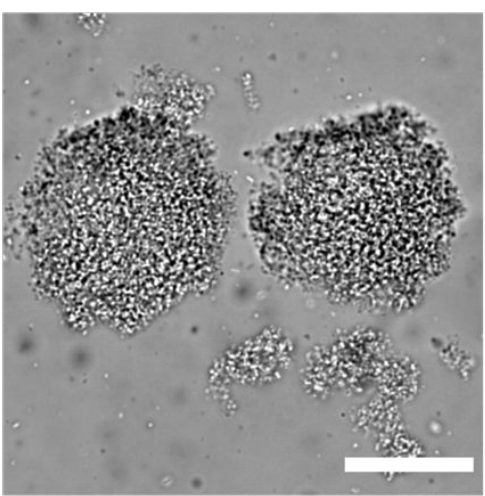

Fluorescent green
Fluorescent magenta
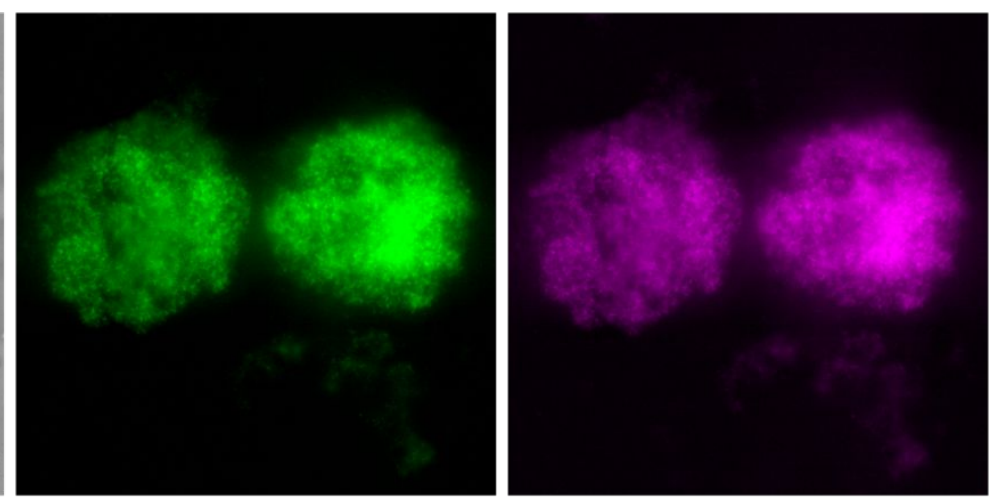

Figure S3. Controls for fluorescent probe (Acridine Orange) staining of E. coli cells. Brightfield and Fluorescent images taken at $50 \times$ show that Acridine Orange $(15 \mu \mathrm{M})$ readily stains non-encapsulated cells. Green and Magenta images depict staining of double and single stranded nucleic acids, respectively. Scale bar $=50 \mu \mathrm{m}$. 


\section{Minimal media $E$. coli growth curves}

Prior to testing the diffusion of sugar molecules within the nanocultures, we performed growth curves for four strains of $E$. coli each in four variations of minimal media, supplemented with 1\% Trace Vitamins (ATCC) and 1\% glucose, the purpose of which was to demonstrate that optimal conditions were achieved for confluent cell growth. The four strains of $E$. coli selected for optimizing cell growth included $E$. coli Nissle wild type (WT), DH5- $\alpha$, mClover (E. coli GFP), and mApple (E. coli RFP). The growth assay contained cells grown in $200 \mu \mathrm{L}$ of minimal media for 48 hours at $37^{\circ} \mathrm{C}$ in aerobic conditions. Absorbance was measured every 10 minutes at $\mathrm{OD}_{600}$ using a 96-well plate reader (Cytation 5 imaging reader, BioTek Instruments Inc.). The growth curves demonstrated that most strains required longer than 24 hours for
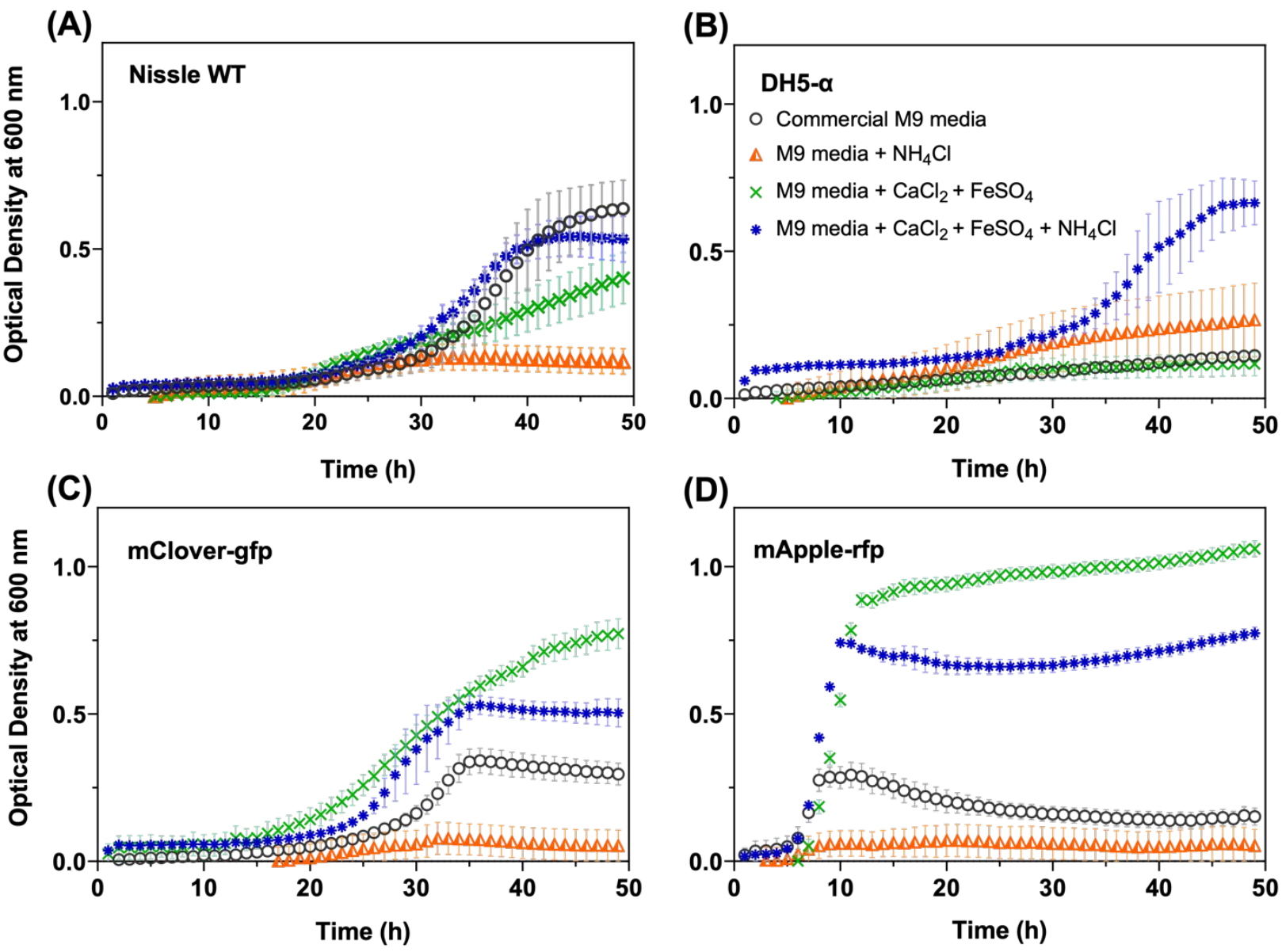

Figure S4. Growth curve optimization of E. coli strains in varying combinations of minimal media. Commercial M9 minimal media was supplemented in stages with $10 \mathrm{mM} \mathrm{NH}_{4} \mathrm{Cl}, 0.1 \% \mathrm{CaCl}_{2}$, and $0.1 \%$ $\mathrm{FeSO}_{4}$. All four versions of minimal media also included $1 \%$ trace vitamins and $1 \%$ glucose. Four $E$. coli strains were tested in the four variations of media, including (A) Nissle WT, (B) DH5- $\alpha$, (C) mClover GFP, and (D) mApple RFP. E. coli mApple RFP performed the best in minimal media supplemented with $\mathrm{CaCl}_{2}$ and $\mathrm{FeSO}_{4}$, reaching approximately $0.8 \mathrm{OD}_{600}$ after 12 hours of growth. 
sufficient growth to be seen, and furthermore, that addition of only $\mathrm{NH}_{4} \mathrm{Cl}$ to minimal media hindered cell growth in most cases (Figure S4). However, E. coli mApple (RFP) demonstrated successful growth within 13 hours when grown in minimal media supplemented with $\mathrm{CaCl}_{2}, \mathrm{FeSO}_{4}, 1 \%$ trace vitamins, and $1 \%$ glucose. Subsequently, the combination of E. coli mApple (RFP) and minimal media supplemented with $\mathrm{CaCl}_{2}, \mathrm{FeSO}_{4}$, and $1 \%$ trace vitamins was selected for the core in nanoculture encapsulation. The test sugar molecules were then added to the external collection solution to determine diffusion.

\section{Diffusion of sugar molecules}

\section{a. Glucose and Sucrose}

After selecting the appropriate strain and minimal media that would ensure cell growth in the presence of a carbon source in the nanocultures, diffusion of glucose and sucrose across the nanoculture membrane was tested as described. In all cases, we observed no cell growth within the nanocultures, observed after 24 hours and again at 48 hours. This indicates that both sugar molecules are impermeable through the nanoculture membrane. Figure S5 show representative images taken at 50× using a Zeiss Axio Imager M2 Epifluorescence and Brightfield Microscope (Carl Zeiss, Inc., Germany).
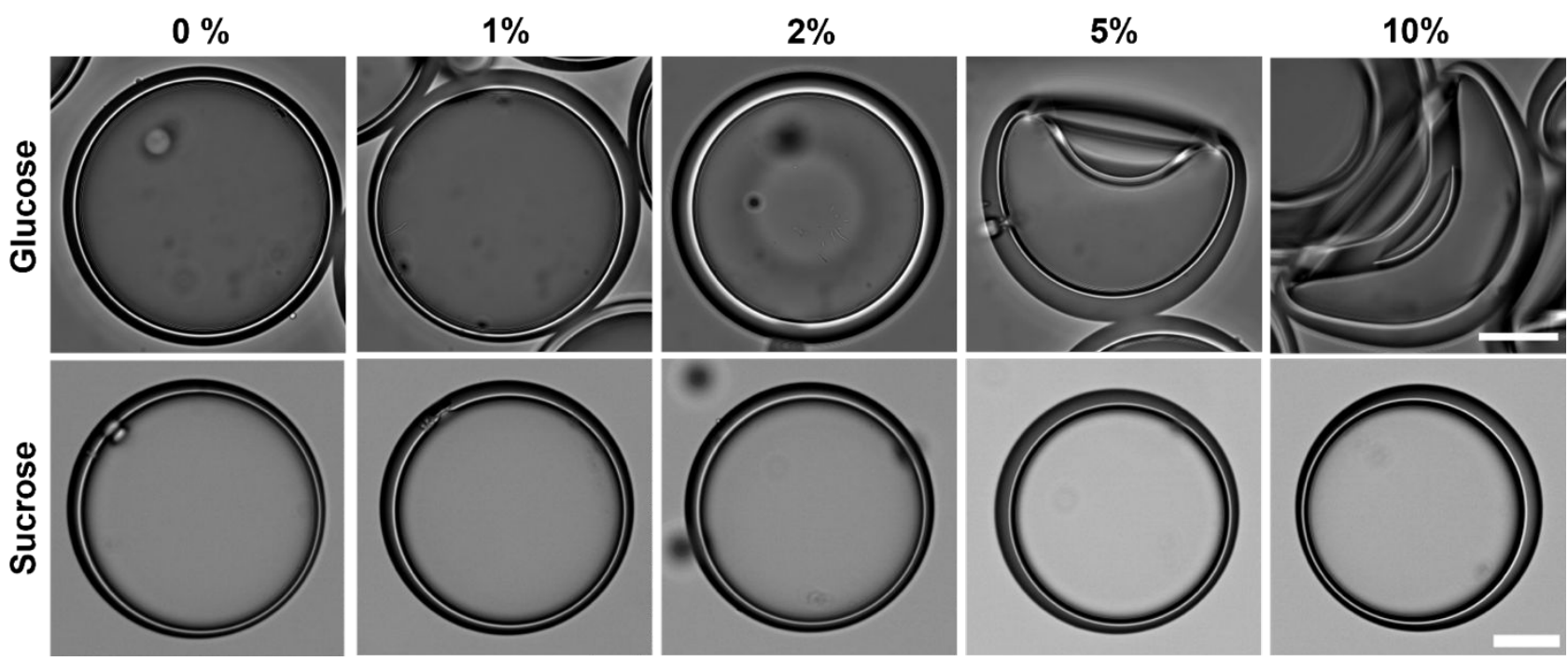

Figure S5. Diffusion of glucose and sucrose molecules through nanocultures membranes. Brightfield imaging showed no cell growth at all, imaged after 24 hours and again at 48 hours. Glucose and sucrose are unable to diffuse through the nanoculture membrane. Images taken at $50 \times$, scale bar $=50$ $\mu \mathrm{m}$. 


\section{b. Arabinose}

To demonstrate the diffusion of arabinose, mean fluorescence intensity was measured within the nanocultures. Positive (100 $\mu \mathrm{g} / \mathrm{mL}$ arabinose) and negative $(0 \mu \mathrm{g} / \mathrm{mL}$ arabinose $)$ controls were included for the comparison of mean fluorescence intensity. In the test sample, $100 \mu \mathrm{g} / \mathrm{mL}$ arabinose was added to the external collection solution. After 24 hours incubation at $37^{\circ} \mathrm{C}$, mean fluorescence intensity was measured and analyzed in software package ImageJ-FIJI. In the positive control, whereby $100 \mu \mathrm{g} / \mathrm{mL}$ arabinose was added to the encapsulated core media, we observed a significantly higher mean fluorescence intensity than both the negative control (no arabinose added to system) and the test sample $(\mathrm{p}<0.0001)$, indicating that arabinose is impermeable through the nanoculture membrane. Figure S5 show representative images taken at 50× using a Zeiss Axio Imager M2 Epifluorescence and Brightfield Microscope (Carl Zeiss, Inc., Germany).
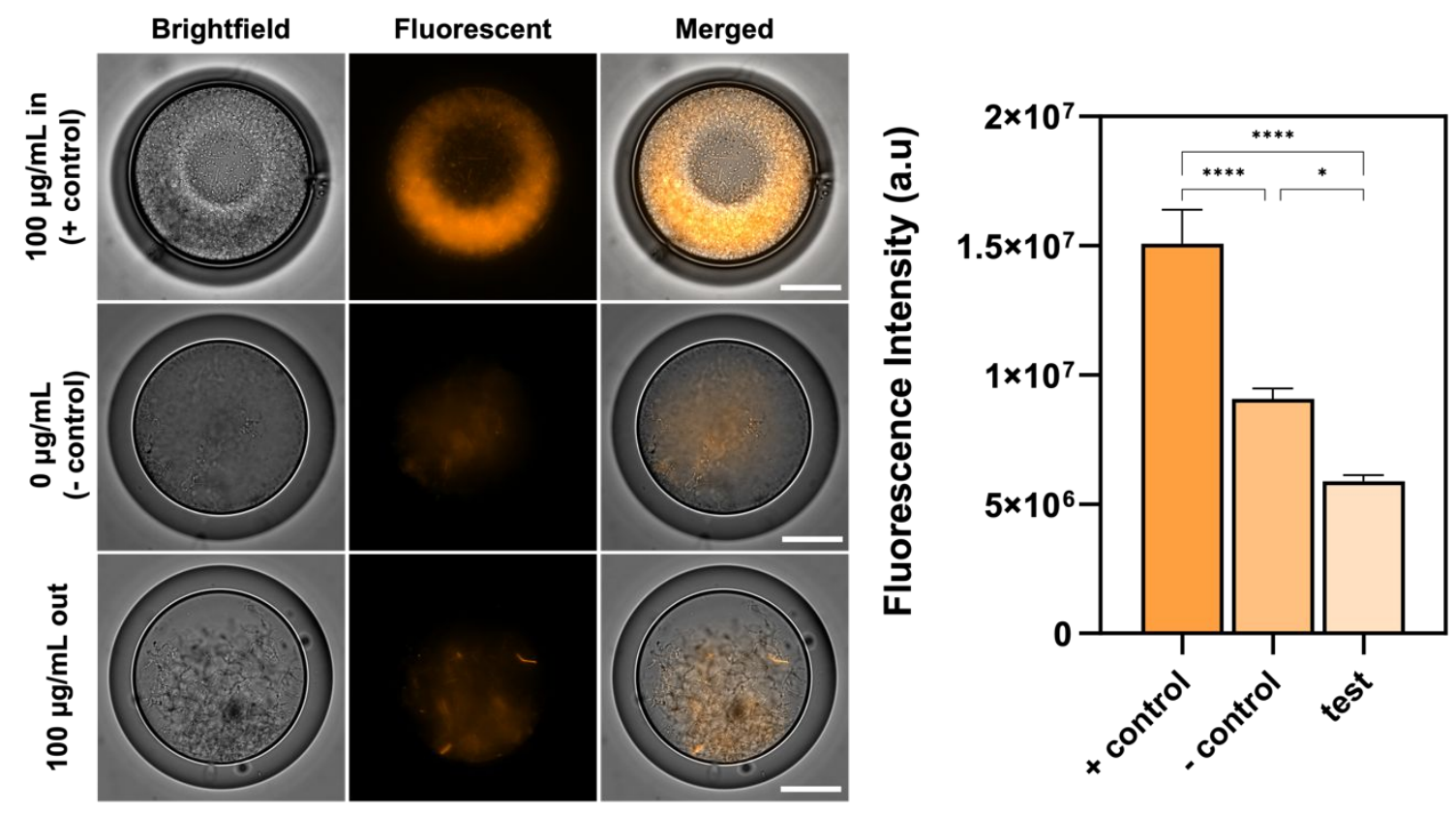

Figure S6. Diffusion of arabinose through nanoculture membrane. Diffusion of arabinose was investigated by proxy of fluorescence intensity of the mApple fluorescent protein which is induced by arabinose. Negative control had no arabinose added $(0 \mu \mathrm{g} / \mathrm{mL})$; baseline fluorescence is due to a leaky promoter. Positive control had $100 \mu \mathrm{g} / \mathrm{mL}$ arabinose added inside the capsule to compare fluorescence. The test sample had $100 \mu \mathrm{g} / \mathrm{mL}$ added externally to the capsule. Positive control had a statistically significant increase in mean fluorescence intensity, indicating that arabinose is impermeable through the nanoculture membrane. One-way ANOVA, Tukey post-hoc. Differences were considered significant when $p<0.05$. **** significant at $p<0.0001$. $*$ Significant at $p=0.0494$. Scale bar $=50 \mu \mathrm{m}$. 


\section{References}

1. Hansen, C. M., Hansen solubility parameters : a user's handbook. 2nd ed. / Charles M. Hansen. ed.; CRC Press: Boca Raton, 2007.

2. Hildebrand, J. H., An Improvement in the Theory of Regular Solutions. Proceedings of the National Academy of Sciences - PNAS 1979, 76 (12), 6040-6041.

3. Lee, J. N.; Park, C.; Whitesides, G. M., Solvent Compatibility of Poly(dimethylsiloxane)-Based Microfluidic Devices. Analytical Chemistry 2003, 75 (23), 6544-6554.

4. Buxton, G. A.; Clarke, N., Drug diffusion from polymer core-shell nanoparticles. Soft Matter 2007, 3 (12), 1513-1517.

5. $\quad$ Knopp, M. M.; Gannon, N.; Porsch, I.; Rask, M. B.; Olesen, N. E.; Langguth, P.; Holm, R.; Rades, T., A Promising New Method to Estimate Drug-Polymer Solubility at Room Temperature. Journal of pharmaceutical sciences 2016, 105 (9), 2621-2624.

6. Mohammad, M. A.; Alhalaweh, A.; Velaga, S. P., Hansen solubility parameter as a tool to predict cocrystal formation. International journal of pharmaceutics 2011, 407 (1), 63-71.

7. Niepa, T. H.; Hou, L.; Jiang, H.; Goulian, M.; Koo, H.; Stebe, K. J.; Lee, D., Microbial Nanoculture as an Artificial Microniche. Sci Rep 2016, 6, 30578.

8. Potter, C. B.; Davis, M. T.; Albadarin, A. B.; Walker, G. M., Investigation of the Dependence of the Flory-Huggins Interaction Parameter on Temperature and Composition in a Drug-Polymer System. Molecular Pharmaceutics 2018, 15 (11), 5327-5335.

9. $\quad$ Flory, P. J., Principles of Polymer Chemistry. Cornell University Press: 1953.

10. Young, R. J.; Lovell, P. A., Introduction to polymers. 3rd ed. ed.; CRC Press: Boca Raton, 2011.

11. Fedors, R. F., A method for estimating both the solubility parameters and molar volumes of liquids. Polymer engineering and science 1974, 14 (2), 147-154.

12. Hoy, K. L., The Hoy tables of solubility parameters. Union Carbide Corporation, Solvents \& Coatings Materials, Research : 1985.

13. Stefanis, E.; Panayiotou, C., Prediction of Hansen Solubility Parameters with a New GroupContribution Method. International journal of thermophysics 2008, 29 (2), 568-585.

14. Van Krevelen, D. W.; Te Nijenhuis, K., Properties of polymers: their correlation with chemical structure; their numerical estimation and prediction from additive group contributions. Elsevier: 2009.

15. Marsac, P. J.; Li, T.; Taylor, L. S., Estimation of Drug-Polymer Miscibility and Solubility in Amorphous Solid Dispersions Using Experimentally Determined Interaction Parameters. Pharmaceutical Research 2008, 26 (1), 139. 